\title{
Exotic Higgs decays at the LHC
}

\author{
Scott Snyder, on behalf of the ATLAS and CMS Collaborations \\ Brookhaven National Laboratory \\ E-mail: snyderabnl.gov
}

\begin{abstract}
Many interesting extensions to the Standard Model predict nonstandard decays of the $125 \mathrm{GeV}$ Higgs boson. The ATLAS and CMS experiments at the LHC have searched for processes including decays of the Higgs boson to long-lived, weakly interacting particles, decays of the Higgs boson to dark photons, decays of the Higgs boson to pairs of new (pseudo)scalars, and leptonflavor violating decays of the Higgs boson. This note will summarize the state of these analyses using data taken through the end of 2016.
\end{abstract}

XXVII International Workshop on Deep-Inelastic Scattering and Related Subjects - DIS2019 8-12 April, 2019

Torino, Italy

${ }^{*}$ Speaker. 


\section{Introduction}

Since the discovery of the Higgs boson in 2012, all measurements of its properties have agreed very well with the expectations of the Standard Model (SM). But there are still open questions for which the Higgs boson could play a major role in the answers, including questions of fine-tuning in the SM, the nature of dark matter, and the origin of the observed matter-antimatter asymmetry. Many models which address these questions predict nonstandard "exotic" decays of the observed $125 \mathrm{GeV}$ Higgs boson [1]. "Exotic" here is not the same as "rare;" in fact, detailed analyses of the couplings of the Higgs boson using Run 1 data imply that the branching fraction of the SM Higgs boson to non-SM particles can be as much as 0.34 (95\% CL) [2], so there is still plenty of room for beyond-SM physics in the decays of the Higgs boson.

This note summarizes four classes of searches: invisible decays of the Higgs boson; Higgs boson decays to a dark-sector photon; decays of the Higgs boson to (pseudo)scalars; and leptonflavor-violating decays of the Higgs boson. These analyses use approximately $36 \mathrm{fb}^{-1}$ of $\sqrt{s}=$ $13 \mathrm{TeV}$ Run 2 data collected through 2016 by the ATLAS and CMS experiments $[3,4]$ at the LHC.

\section{Invisible decays of the Higgs boson}

Many extensions to the SM predict decays of the SM Higgs boson into weakly-interacting massive particles (WIMPs), which can be invisible to the detector. As the $H \rightarrow 4 v$ rate in the SM is quite small, this is a promising way to search for new physics.

The Higgs boson could be produced by vector-boson fusion (VBF); in this case, one looks for two jets widely separated in $\eta$ with large $m_{j j}$. One can also look for a Higgs boson produced in association with either a $Z$ boson decaying to a pair of leptons or a $W / Z$ boson decaying to a pair of quarks; in the latter case, one can look for both final states with two resolved jets and final states in which the two jets have merged into a single large jet, consistent with $W / Z$ boson decay. This final state will also have sensitivity to the case where a Higgs boson is produced recoiling against a jet. In all cases, one also requires a large $E_{\mathrm{T}}^{\mathrm{miss}}$, ascribed to the invisible Higgs boson decay. Both ATLAS and CMS have carried out searches in these channels [5-8]; selected distributions from these analyses are shown in Figure 1. Limits are extracted by fitting the distributions of either $m_{j j}$ in the VBF case, or $E_{\mathrm{T}}^{\text {miss }}$ otherwise; the CMS analysis with $Z \rightarrow \ell \ell$ uses a boosted decision tree (BDT) to extract the limit. The resulting limits on the branching fraction of invisible decays of the Higgs boson $\mathcal{B}_{\text {inv }}$, along with combinations over data taken through 2016, are (at 95\% CL with expected limits in parentheses):

\begin{tabular}{lll} 
Channel & ATLAS $\mathcal{B}_{\text {inv }}$ & CMS $\mathcal{B}_{\text {inv }}$ \\
VBF $(H \rightarrow$ inv $)$ & $<0.37(0.28)[8]$ & $<0.33(0.25)[5]$ \\
$(H \rightarrow$ inv $)+(Z \rightarrow \ell \ell)$ & $<0.67(0.39)[8]$ & $<0.40(0.42)[6]$ \\
$(H \rightarrow$ inv $)+(W / Z \rightarrow$ had $)$ & $<0.83(0.58)[8]$ & $<0.53(0.40)[7]$ \\
\hline Run 1 $\left(25 \mathrm{fb}^{-1}, 7+8 \mathrm{TeV}\right)$ & $<0.25(0.27)[8]$ & \\
Run $1+2015\left(27.1 \mathrm{fb}^{-1}, 7+8+13 \mathrm{TeV}\right)$ & & $<0.24(0.23)[9]$ \\
$2015+2016\left(36.1 \mathrm{fb}^{-1}\right)$ & $<0.38(0.21)[8]$ & \\
$2016\left(35.9 \mathrm{fb}^{-1}\right)$ & & $<0.26(0.20)[5]$ \\
\hline All & $<0.26(0.17)[8]$ & $<0.19(0.15)[5]$
\end{tabular}


Figure 1 also shows the CMS results interpreted as a limit on nuclear scattering cross section as a function of the WIMP mass, given one of two specific models (comparable ATLAS results are in [8]). Compared to limits from direct-detection experiments, it is seen that the LHC results contribute significantly for small WIMP masses (between 1 and $10 \mathrm{GeV}$ ).
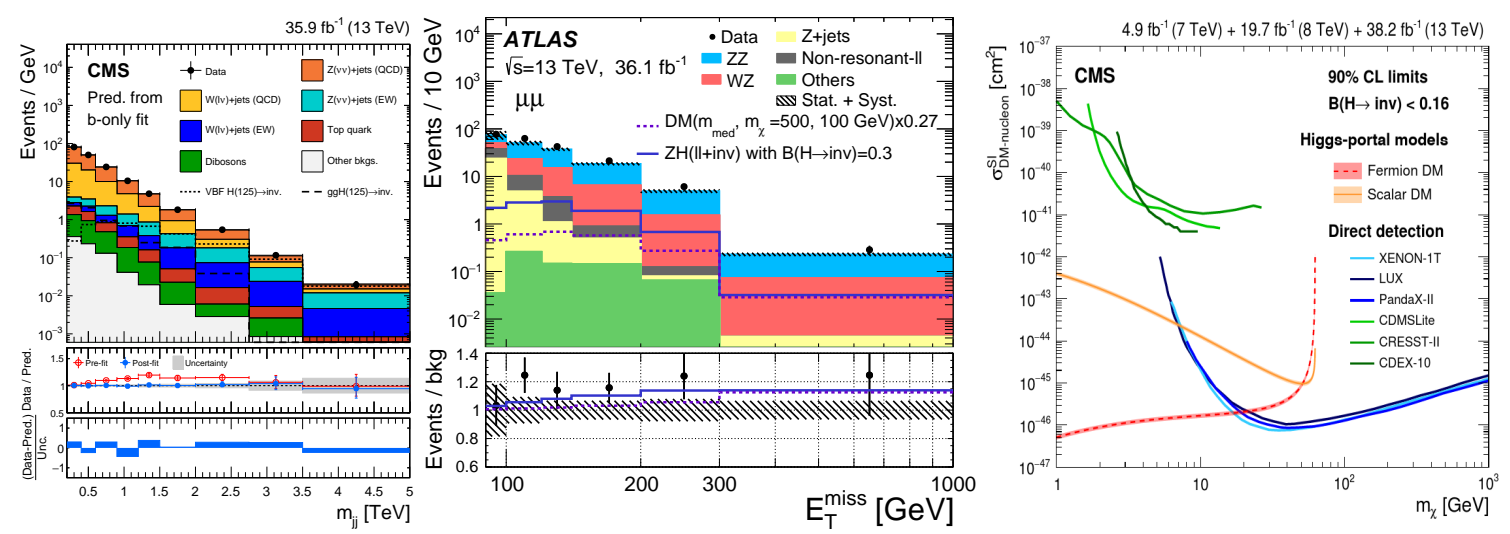

Figure 1: Selected $H \rightarrow$ inv search results. Left: $m_{j j}$ distribution from the CMS VBF $H \rightarrow$ inv search [5]; Middle: $E_{\mathrm{T}}^{\text {miss }}$ distribution from the ATLAS $(H \rightarrow$ inv $)(Z \rightarrow \ell \ell)$ search in the $\mu \mu$ channel [10]; Right: CMS nuclear scattering cross section limits for specific WIMP models compared to direct-detection limits [5].

\section{Higgs boson decays to dark photons}

Some dark-matter-inspired extensions to the SM include a $U(1)$ dark gauge symmetry, giving rise to a gauge boson $Z_{d}$, or "dark photon" [11]. This dark photon can then mix with the SM Higgs boson with some strength $\kappa$, giving rise to a $H \rightarrow Z_{d} Z_{d}$ decay; or it could mix with the SM gauge bosons with strength $\varepsilon$, giving rise to $H \rightarrow Z Z_{d}$ decays. The $Z_{d}$ should decay to lepton pairs with a significant branching fraction $(\sim 15 \%)$; these decays will be prompt for $\varepsilon \gtrsim 10^{-5}$.

ATLAS has searched for these processes in $4 \ell$ final states [12] consisting of two lepton pairs: $4 e, 2 e 2 \mu$, and $4 \mu$. The overall invariant mass must be consistent with the SM Higgs boson mass. For $H \rightarrow Z_{d} Z_{d}$, each lepton pair must be inconsistent with both $J / \psi / \Upsilon$ decays and with mispaired $Z$ boson decay, and the two lepton pairs must have similar mass. For the low-mass region $1<$ $m_{Z_{d}}<15 \mathrm{GeV}$, only the $4 \mu$ final state is considered. Backgrounds are primarily $H \rightarrow Z Z^{*}$ and $Z Z^{*}$. In the high (low)-mass region, $6(0)$ events are observed with an expected background of $3.9 \pm 0.3(0.4 \pm 0.1)$. For $H \rightarrow Z Z_{d}$, one lepton pair must be consistent with $Z \rightarrow \ell \ell$ decay, and one looks for a peak in the invariant mass of the other pair. Here, 102 events are observed, with a background of $86.8 \pm 7.5$. Selected results are shown in Figure 2.

\section{Higgs boson decays to scalars}

Models with two Higgs doublets and an additional scalar field (2HDM+S) predict that the SM Higgs boson can decay into a new (pseudo)scalar $a$ [1]. The $a \rightarrow b b$ decay would usually dominate, but other decays may be significant depending on the model, such as $a \rightarrow \mu \mu, \tau \tau, \gamma \gamma$, or $g g$.

ATLAS has searched for such decays in several final states. (The corresponding CMS analyses are covered in [13] and are not repeated here.) First $(V \rightarrow \ell \ell / \ell v)(H \rightarrow a a \rightarrow b b b b)$ [14], requiring 

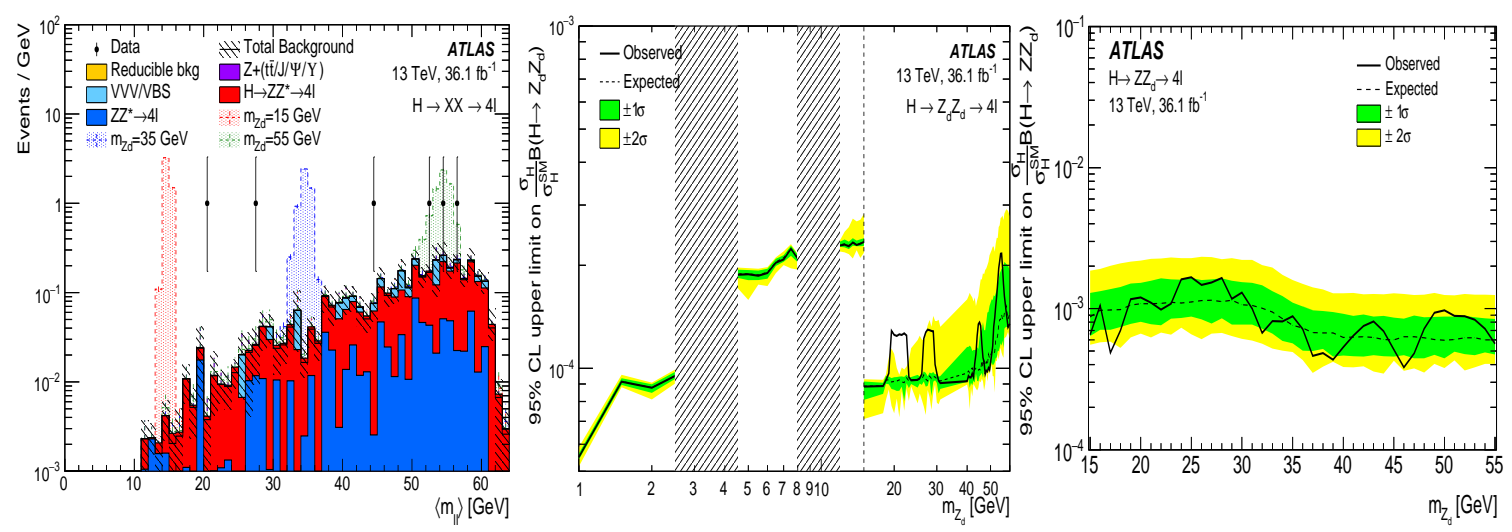

Figure 2: Selected results from the ATLAS dark photon searches [12]. Left: Average invariant mass of the two lepton pairs in the $H \rightarrow Z_{d} Z_{d}$ search for the high-mass region; Middle: 95\% CL limits on $\mathcal{B}\left(H \rightarrow Z_{d} Z_{d}\right)$ as a function of $Z_{d}$. The gray strips are regions excluded from the analysis due to large backgrounds from $J / \psi$ and $\Upsilon$ decays; Right: $95 \%$ CL limits on $\mathcal{B}\left(H \rightarrow Z Z_{d}\right)$ as a function of $Z_{d}$.

a reconstructed, leptonically-decaying $W$ or $Z$ boson along with at least three $b$-tagged jets. Jets are paired to minimize the difference in invariant masses between the pairs, and BDTs are used to discriminate signal from background. Second, $H \rightarrow a a \rightarrow b b \mu \mu$ [15], significant in some 2HDM models with enhanced lepton couplings. This requires a $\mu^{+} \mu^{-}$pair with $16<m_{\mu \mu}<64 \mathrm{GeV}$, exactly two $b$-tagged jets, $E_{\mathrm{T}}^{\text {miss }}<60 \mathrm{GeV}$, and that the event is kinematically consistent with a decay of the $125 \mathrm{GeV}$ SM Higgs boson. Third, $H \rightarrow a a \rightarrow \gamma \gamma g g$ [16], significant in models in which decays to fermions are suppressed. The production of the Higgs boson is tagged by the presence of two VBF jets, and again the event must be kinematically consistent with the decay hypothesis. Selected results from these analyses are shown in Figure 3, which also shows limits on $\mathcal{B}(H \rightarrow a a)$ from all ATLAS $H \rightarrow a a$ searches under the assumption of a specific 2HDM+S model [17], including also results from the $H \rightarrow Z_{d} Z_{d}$ analysis reinterpreted as $H \rightarrow a a$.

\section{Lepton-flavor-violating Higgs boson decays}

Decays of the Higgs boson into two leptons of differing flavor are forbidden in the SM, but are allowed in some extensions, including supersymmetry, composite Higgs, Randall-Sundrum models, and others (see references in [18]). The decay $H \rightarrow e \mu$ is very strongly constrained from searches for $\mu \rightarrow e \gamma$ to $\mathcal{B}(H \rightarrow e \mu)<\mathcal{O}\left(10^{-9}\right)$; however, limits on $H \rightarrow e \tau$ and $H \rightarrow \mu \tau$ from searches for rare $\tau$ decays are much larger, $\mathcal{O}(10 \%)$ [18].

CMS has searched for the decays $H \rightarrow e \tau$ and $H \rightarrow \mu \tau$, where the $\tau$ lepton decays either hadronically or into a lepton of opposite flavor [18]. This gives four channels: $e \tau_{h}, e \tau_{\mu}, \mu \tau_{h}$, and $\mu \tau_{e}$. Each of these is then subdivided into categories based on the jets in the event: 0 jets, 1 jet, 2-jet VBF, and other 2-jet events ( $\mathrm{ggH})$. The signal is discriminated using BDTs, and the limit is extracted via a joint fit to the BDT outputs. Backgrounds are primarily $Z \rightarrow \tau \tau, W+$ jets, and QCD with jets misidentified as leptons. Sample results from this analysis are shown in Figure 4. Results, at $95 \% \mathrm{CL}$, are $\mathcal{B}(H \rightarrow \mu \tau)<0.25(0.25) \%$ and $\mathcal{B}(H \rightarrow e \tau)<0.61(0.37) \%$. 

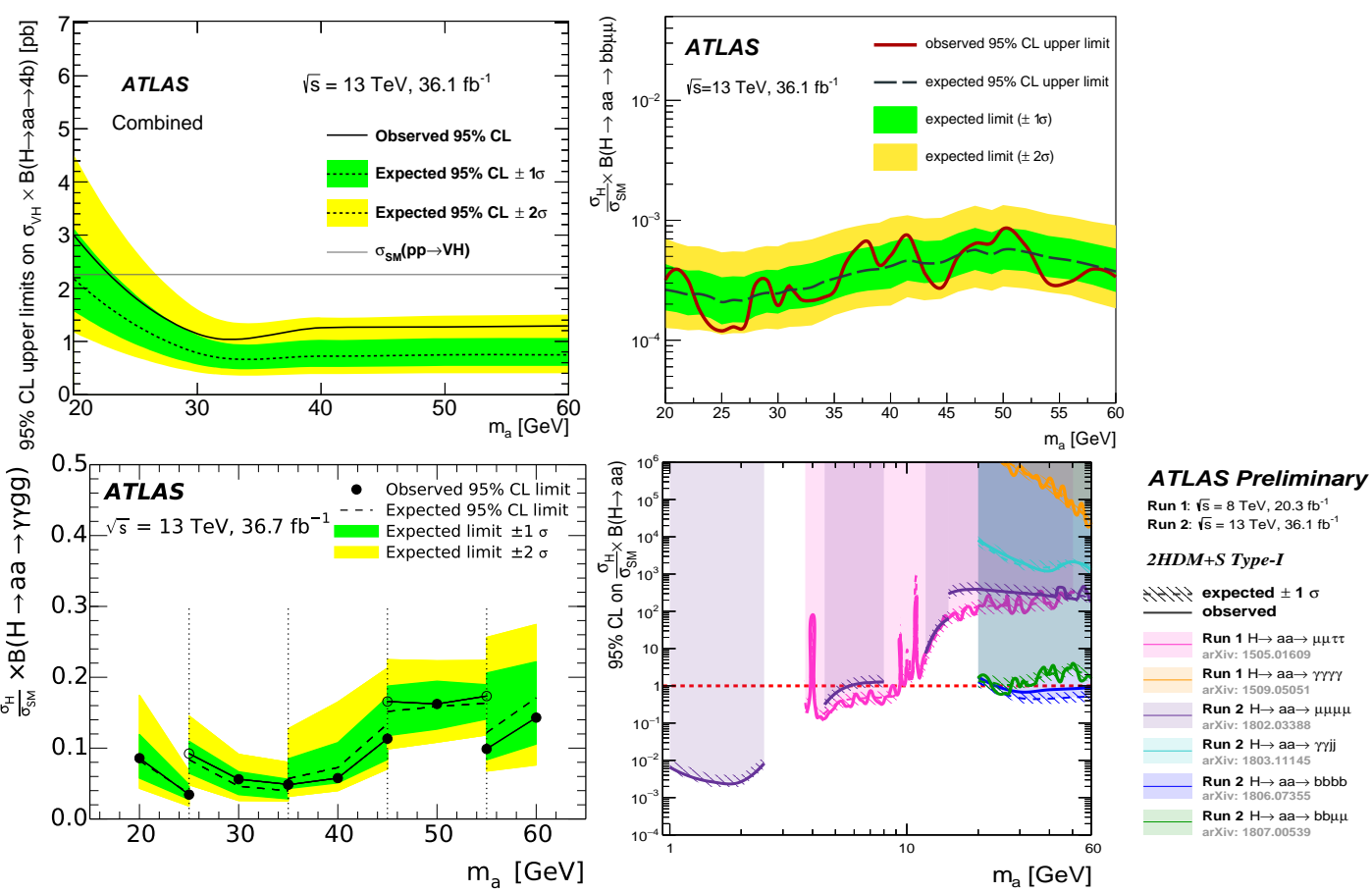

Figure 3: Selected results from the ATLAS searches for Higgs boson decays to new scalars. Top left: $95 \%$ CL limit for $\mathcal{B}(H \rightarrow a a \rightarrow b b b b)$ from $V H$ production [14]; Top right: 95\% CL limits on $\mathcal{B}(H \rightarrow a a \rightarrow$ $b b \mu \mu)$ [15]; Bottom left: 95\% CL limit for $\mathcal{B}(H \rightarrow a a \rightarrow \gamma \gamma g g)$ [16]; Bottom right: 95\% CL limits on $H \rightarrow a a$ from all ATLAS searches assuming a Type-I 2HDM+S model [17].

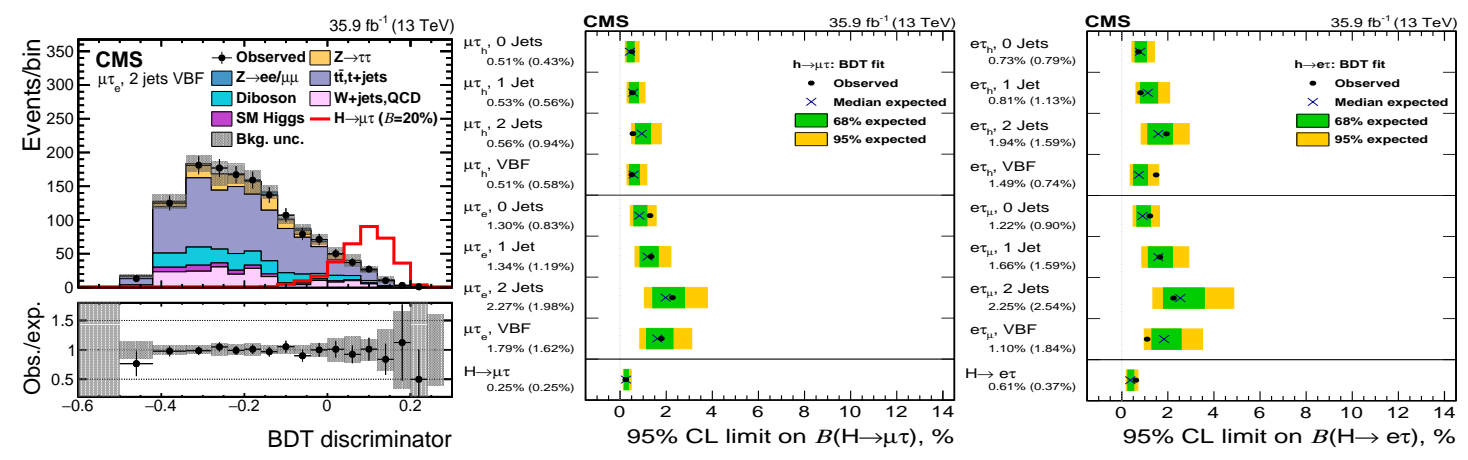

Figure 4: Selected results from the CMS search for lepton-flavor violating decays of the Higgs boson [18]. Left: BDT output for the $\mu \tau_{e}$ channel with 2 VBF jets; Middle: Limits on $\mathcal{B}(H \rightarrow \mu \tau)$ per channel and category; Right: Limits on $\mathcal{B}(H \rightarrow e \tau)$ per channel and category.

\section{Summary}

Nonstandard decays of the SM Higgs boson, predicted by many extensions to the SM, have been searched for by ATLAS and CMS at the LHC. No excesses have been found in data taken through 2016, and limits have been set on branching fractions for the exotic decays $H \rightarrow$ invisible, $H \rightarrow Z Z_{d} / Z_{d} Z_{d}, H \rightarrow a a$, and $H \rightarrow e \tau / \mu \tau$.

This work is supported in part by the U.S. Department of Energy under contract DE-AC0298CH10886 with Brookhaven National Laboratory. 


\section{References}

[1] D. Curtin et al., Exotic decays of the 125 GeV Higgs boson, Phys. Rev. D90 (2014) 075004.

[2] ATLAS and CMS Collaborations, Measurements of the Higgs boson production and decay rates and constraints on its couplings from a combined ATLAS and CMS analysis of the LHC pp collision data at $\sqrt{s}=7$ and $8 \mathrm{TeV}, \mathrm{JHEP} 08$ (2016) 045 [1606.02266].

[3] ATLAS Collaboration, The ATLAS Experiment at the CERN Large Hadron Collider, JINST 3 (2008) S08003.

[4] CMS Collaboration, The CMS experiment at the CERN LHC, JINST 3 (2008) S08004.

[5] CMS Collaboration, Search for invisible decays of a Higgs boson produced through vector boson fusion in proton-proton collisions at $\sqrt{s}=13 \mathrm{TeV},(2018)$ [1809.05937].

[6] CMS Collaboration, Search for new physics in events with a leptonically decaying $Z$ boson and $a$ large transverse momentum imbalance in proton-proton collisions at $\sqrt{s}=13 \mathrm{TeV}$, Eur. Phys. J. C 78 (2018) 291 [1711.00431].

[7] CMS Collaboration, Search for new physics in final states with an energetic jet or a hadronically decaying $W$ or $Z$ boson and transverse momentum imbalance at $\sqrt{s}=13 \mathrm{TeV}$, Phys. Rev. D 97 (2018) 092005 [1712.02345].

[8] ATLAS Collaboration, "Combination of searches for invisible Higgs boson decays with the ATLAS experiment.” ATLAS-CONF-2018-054, 2018. https://cds.cern.ch/record/2649407.

[9] CMS Collaboration, Searches for invisible decays of the Higgs boson in pp collisions at $\sqrt{s}=7,8$, and $13 \mathrm{TeV}, \mathrm{JHEP} 02$ (2017) 135 [1610.09218].

[10] ATLAS Collaboration, Search for an invisibly decaying Higgs boson or dark matter candidates produced in association with a $Z$ boson in pp collisions at $\sqrt{s}=13 \mathrm{TeV}$ with the ATLAS detector, Phys. Lett. B 776 (2018) 318 [1708.09624].

[11] D. Curtin, R. Essig, S. Gori and J. Shelton, Illuminating Dark Photons with High-Energy Colliders, JHEP 02 (2015) 157 [1412.0018].

[12] ATLAS Collaboration, Search for Higgs boson decays to beyond-the-Standard-Model light bosons in four-lepton events with the ATLAS detector at $\sqrt{s}=13 \mathrm{TeV}, \mathrm{JHEP} 06$ (2018) 166 [1802.03388].

[13] A. Kalogeropoulos, Searches for Light Higgs Bosons at the CMS Experiment, in Proceedings of DIS2019, POS (DIS2019) 096, (2019).

[14] ATLAS Collaboration, Search for the Higgs boson produced in association with a vector boson and decaying into two spin-zero particles in the $H \rightarrow a a \rightarrow 4 b$ channel in pp collisions at $\sqrt{s}=13 \mathrm{TeV}$ with the ATLAS detector, JHEP 10 (2018) 031 [1806.07355].

[15] ATLAS Collaboration, Search for Higgs boson decays into a pair of light bosons in the bb $\mu \mu$ final state in pp collision at $\sqrt{s}=13$ TeV with the ATLAS detector, Phys. Lett. B 790 (2019) 1 [1807.00539].

[16] ATLAS Collaboration, Search for Higgs boson decays into pairs of light (pseudo)scalar particles in the $\gamma \gamma j$ j final state in pp collisions at $\sqrt{s}=13$ TeV with the ATLAS detector, Phys. Lett. B 782 (2018) $750[1803.11145]$.

[17] ATLAS Collaboration, "HBSM Working Group 2HDM+S Summary Plots." ATL-PHYS-PUB-2018-045, 2018. https://cds.cern.ch/record/2650740.

[18] CMS Collaboration, Search for lepton flavour violating decays of the Higgs boson to $\mu \tau$ and $e \tau$ in proton-proton collisions at $\sqrt{s}=13 \mathrm{TeV}$, JHEP 06 (2018) 001 [1712.07173]. 